\title{
Lista de especies cinegéticas en tres sectores del parque Nacional Natural Alto Fragua Indi Wasi, Caquetá, Colombia
}

\author{
List of Wildlife hunting species in three sectors of The Alto Fragua Indi \\ Wasi National Natural Park, Caquetá, Colombia
}

Morales-Collazos MA. Lista de especies cinegéticas en tres sectores del parque Nacional Natural Alto Fragua Indi Wasi, Caquetá; Colombia. Rev Colombiana Cienc Anim. Recia. 2020; 12(2):e764. https://doi.org/10.24188/recia.v12.n2.2020.764

Universidad de Sucre, Colombia

Los autores permiten a RECIA reimprimir el material publicado en él. En caso de que un autor quiera traducir o usar una publicación parcial o completa de nuestro Diario, el autor debe obtener un permiso por escrito del editor de la revista.

Copyright (C) 2020. El (los) autor (es), Revista Colombiana de Ciencia Animal - RECIA. 2020. Este es un artículo de acceso abierto distribuido bajo los términos de Creative Commons Attribution 4.0 (https://creativecommons.org/licenses/by-nc-sa/4.0/), El uso, distribución o reproducción está permitido, siempre que se acrediten al autor original y al propietario del copyright y que se cite la publicación original en esta revista, de acuerdo con la práctica académica aceptada. No se permite el uso, distribución o reproducción que no cumpla con estos términos. 


\title{
Lista de especies cinegéticas en tres sectores del parque Nacional Natural Alto Fragua Indi Wasi, Caquetá, Colombia
}

\author{
List of Wildlife hunting species in three sectors of The Alto Fragua Indi Wasi National \\ Natural Park, Caquetá, Colombia
}

Mario Alberto Morales Collazos. M.Sc.

DOI: https://doi.org/10.24188/recia.v12.n2.2020.764

Universidad del Cauca. Fundación para el Manejo y Conservación de la Biodiversidad Colombiana - BIOTELLUS, Grupo de Estudios en Geología, Ecología y Conservación - GECO. Colombia. biomarioal@gmail.com

(Dhttps://orcid.org/0000-0003-3348-0162

Recepción: 15 abril 2020

Aprobación: 18 julio 2020

Publicación: 4 agosto 2020

\section{RESUMEN}

Con el objetivo de brindar una información base de fauna cinegética en comunidades campesinas en el piedemonte amazónico, se presenta una lista de especies registradas en tres sectores del Parque Nacional Natural Alto Fragua Indi Wasi en el departamento del Caquetá: Fragua Grande, Fragua Chorroso y Pescado, obtenida en el marco de la caracterización de de cacería de fauna silvestre que se desarrolló entre los meses de abril y diciembre de 2018. La lista se obtuvo de la realización de 43 entrevistas semiestructuradas a las comunidades campesinas y observaciones personales de la actividad. Se registró un total de 20 especies de uso cinegético pertenecientes a la clase Mammalia (17 especies) y Aves (tres especies). De las especies registradas se encontró que tres de ellas están en categoría vulnerables (VU) y una en estado crítico (CR) según la resolución 1912 del 2017 para Colombia y según la clasificación mundial de la IUCN, 13 estuvieron en preocupación menor (LC), cinco en estado vulnerable (VU) y una cerca a la amenaza (NT). La lista presentada y su estado de conservación aportan al manejo de la fauna cinegética dentro del área protegida.

Palabras Clave: Cacería; fauna silvestre; piedemonte amazónico; Caquetá; campesinos.

\begin{abstract}
With the aim of providing an information baseline about wild life hunting of peasant communities in the Amazon foothills, a list of species evaluated in three sectors of the Alto Fragua Indi Wasi National Natural Park in the department of Caquetá is presented: Fragua Grande, Fragua Chorroso and Pescado, obtained within the framework of the characterization of wildlife hunting activity that was developed between the months of April and December 2018. The list was obtained of 43 semi-structured interviews that were carried out with peasant communities and personal observations of the activity. A total of 20 species of cinegetic use belonging to the classes Aves (3 species) and Mammalia (17 species) were registered. Of the registered species it was found that three of them are in vulnerable state (VU), one in critical state (CR) according to the Resolution 1912 of 2017 for Colombia were reported and according to the IUCN classification, 13 of them were at lower critical (LC), 5 of them in a vulnerable state (VU) and one was nearly-threatened (NT). The list presented and its state of conservation contribute to the management of wild life hunting within protected area.
\end{abstract}

Keywords: Hunting, Wildlife, Amazon Foothill, Caquetá, peasants. 


\section{INTRODUCCIÓN}

La fauna silvestre es un recurso natural renovable subestimado que no es comparable con los recursos pesqueros o forestales, a pesar de tener muchos usos (1) y, en referencia a todos aquellos animales no domésticos (2), es el recurso principal de carne para cientos de millones de personas a nivel mundo (3).

La fauna cinegética son todos los animales vertebrados, con excepción de los peces, que son objeto de caza y que aportan a las necesidades de las comunidades humanas, principalmente como suplemento alimenticio, pero así mismo, son utilizados como medicina, para rituales culturales, objetos de comercio, animales de compañía (3).

La fauna amazónica es un patrimonio natural importante, por la diversidad de especies que la representa, las funciones ecológicas que cumple en los ecosistemas amazónicos y por su aporte como recurso económico y de alimento de la gente que habita una región. Es preocupante, sin embargo, que la presión de caza en muchas regiones convierta su extracción en una práctica insostenible (4) lo que ha llevado a muchas especies al borde de la extinción.

En el Parque Nacional Natural Alto Fragua Indi Wasi (PNNAFIW) existen poblaciones de fauna silvestre que son usadas por las comunidades campesinas que habitan al interior del área protegida o en zonas de traslape, lo que ha permitido que la dirección del PNNAFIW haya realizado algunas jornadas de campo para recopilar información base sobre la actividad de cacería. Teniendo en cuenta que la cacería de fauna silvestre es una de las amenazas identificada en el Plan de Manejo Ambiental del área protegida, fue importante conocer cuáles especies son cazadas por las comunidades campesinas en tres sectores en las cuencas de los ríos Fragua Grande, Fragua Chorroso y Pescado,

La lista de especies generada será util en el manejo de la fauna silvestre y en el desarrollo de las estrategias de mitigación de impactos que se incluyen en el plan de manejo del área protegida, además, permite un punto de comparación con listados que se han generado con comunidades indígenas, lo cuál representa una apropiación e interpretación territorial por cada uno de éstos grupos humanos en el pie de monte amazónico.

\section{MATERIALES Y MÉTODOS}

Área de estudio. El estudio se realizó en el PNNAFIW (Figura 1), localizado en el flanco oriental de la cordillera oriental de los Andes colombianos, en jurisdicción de los municipios de San José del Fragua y Belén de los Andaquíes, departamento del Caquetá. El PNNAFIW limita al occidente con el Parque Nacional Natural Churumbelos Auka Wasi, al norte con el Parque Nacional Natural Cueva de los Guacharos y la Reserva Forestal de la Amazonía, al oriente y sur con Belén de los Andaquíes, San José del Fragua y la carretera marginal de la Selva (Plan de manejo PNNAFIW 2012). El estudio se realizó en los sectores de manejo Fragua Grande, Fragua Chorroso y Pescado.

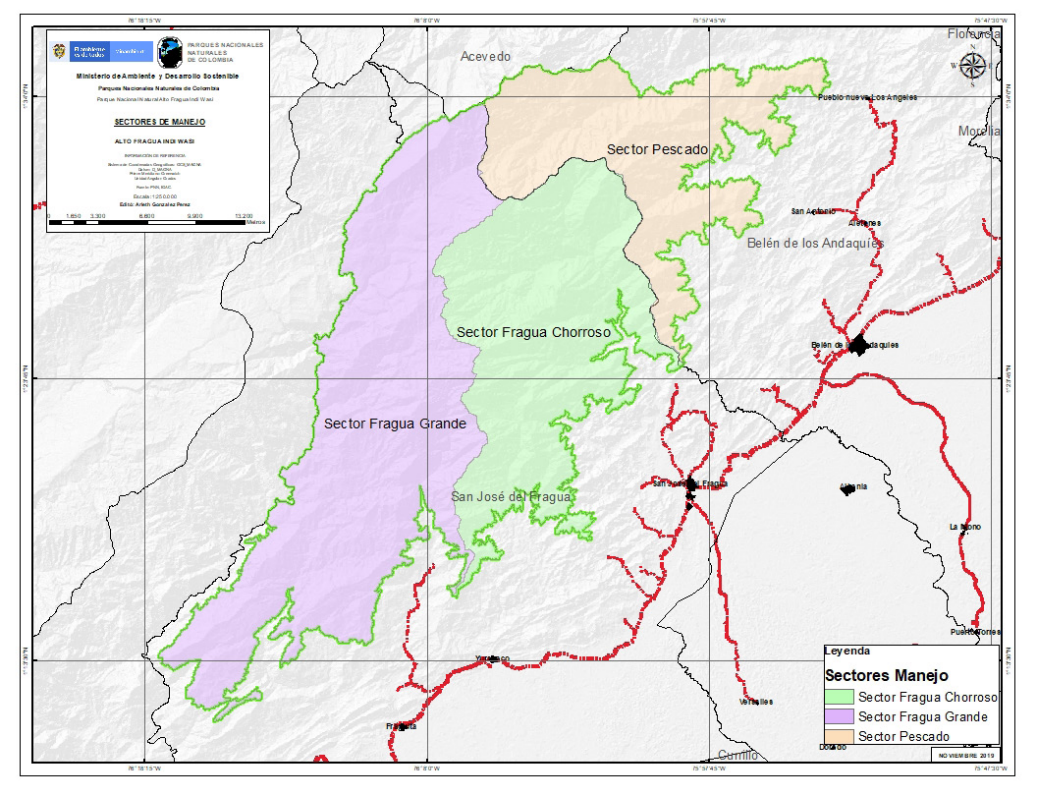

Figura 1. Mapa general de los sectores de estudio. PNNAFIW. Fuente: Parque Nacional Natural Alto Fragua Indi Wasi. 
Recolección de Información: en el marco del proyecto de investigación Caracterización de la actividad de cacería de fauna silvestre en tres sectores del PNNAFIW (2018 - 2019), se realizó una revisión de información secundaria institucional (años 2009 y 2012) que abordaran el tema de la cacería al interior del parque y zonas limítrofes, con el fin de conocer la información de contexto para el diseño de encuestas semiestructuradas para la comunidad de campesinos.

Se realizaron un total de 43 encuestas semiestructuradas entre marzo y noviembre del 2018 en los sectores Fragua Chorroso, Fragua Grande y Pescado, en las cuales se buscó obtener, de modo sistemático y no intrusivo, datos sobre las especies de fauna silvestre que son cazadas, algunos de sus usos y la localización espacial de la actividad.

De esta información se extrajo la necesaria para la construcción de la lista de especies con potencial cinegético y se enfatizó sobre el estado de conservación de las especies con más riesgo, ya que aporta al manejo de la fauna cinegética por parte del PNNAFIW en articulación con su plan de manejo y sus valores objeto de conservación. Para garantizar que los comuneros identificaran las especies se usaron láminas de fauna silvestre y huellas.

\section{RESULTADOS}

La comunidad campesina reportó un total de 20 especies para los tres sectores del PNNAFIW, pertenecientes a la clase Aves (tres especies) y a la clase Mammalia (17 especies) (Tabla 1).

Tabla 1. Lista de especies de fauna silvestre cinegéticas en tres sectores del PNNAFIW.

\begin{tabular}{|c|c|c|c|c|c|}
\hline Clase & Familia & Especie & Nombre Común & E1 & E2 \\
\hline Mammalia & Canidae & Cerdocyon thous & Zorro perro & sin reporte & LC \\
\hline Mammalia & Erethizontidae & Coendou prehensilis & Puerco espín & sin reporte & LC \\
\hline Aves & Cracidae & Crax sp & Paujil & no aplica & no aplica \\
\hline Mammalia & Cuniculidae & Cuniculus paca & Boruga & sin reporte & LC \\
\hline Mammalia & Dasyproctidae & Dasyprocta fuliginosa & Coatín & sin reporte & LC \\
\hline Mammalia & Dasypodidae & Dasypus C.f. novemcinctus & Armadillo & sin reporte & LC \\
\hline Mammalia & Caviidae & Hydrochoerus hydrochaeris & Chiguiro & sin reporte & LC \\
\hline Mammalia & Atelidae & Lagothrix lagotricha & Mico churuco & $\mathrm{VU}$ & $\mathrm{VU}$ \\
\hline Mammalia & Felidae & Leopardus tigrinus & Tigrillo & VU & VU \\
\hline Mammalia & Myrmecophagidae & Myrmecophaga tridactyla & Oso palmero & VU & VU \\
\hline Mammalia & Dasyproctidae & Myoprocta pratti & Tintín & sin reporte & LC \\
\hline Mammalia & Procyonidae & Nasuella olivacea & Cuzumbo & sin reporte & NT \\
\hline Mammalia & Cervidae & Odocoileus virginianus & Venado & sin reporte & LC \\
\hline Aves & Cracidae & Ortalis guttata & Guacharaca & sin reporte & LC \\
\hline Mammalia & Tayassuidae & Pecari tajacu & Manao & sin reporte & LC \\
\hline Aves & Cracidae & Penelope jacquacu & Torcaza & sin reporte & LC \\
\hline Mammalia & Procyonidae & Potos flavus & Perro de monte & sin reporte & LC \\
\hline Mammalia & Cebidae & Sapajus apella & Mico maicero & sin reporte & LC \\
\hline Mammalia & Tapiridae & Tapirus terrestris & Danta & CR & $\mathrm{VU}$ \\
\hline Mammalia & Tayassuidae & Tayassu pecari & Cerrillo & Sin reporte & VU \\
\hline
\end{tabular}

E1= Estado de Conservación Nacional (Resolución 1912 del 2017) ; E2= Estado de Conservación IUCN Internacional.

A continuación, se relacionan las especies para cada uno de los sectores evaluados:

Sector Fragua Grande. Para éste sector se tuvo un registro de 13 especies pertenecientes al grupo de los mamíferos; la mayor representación la tuvieron la guagua y el armadillo con un $18.75 \%$ (el porcentaje a la frecuencia de reporte en las encuestas) cada una, seguida del venado cola blanca y el cerrillo con un $9.38 \%$ cada una y el coatín, el cuzumbo, el manao y la guacharaca con un 6.25\% cada una; se registró igualmente al paujil (Crax sp), el chiguiro (Hydrochoerus hydrochaeris), el mico churuco (Lagothrix lagotricha), el oso palmero (Myrmecophaga tridactyla) y el mico maicero (Sapajus apella). 
Sector Fragua Chorroso. En este sector se registró un total de ocho especies pertenecientes a la clase Mammalia. La mayor representación la tuvo el armadillo (30.76\%), seguido por la guagua (26.92\%) y el coatín (11.53\%). Se registraron igualmente el cuzumbo, el zorro perro, el mico churuco, el tigrillo y el cerrillo.

Sector Pescado. En este sector se registró un total de 16 especies pertenecientes al grupo de las aves y los mamíferos; la mayor representación la tuvo la guagua con un $25.47 \%$, seguida por el armadillo con un $23.58 \%$ y el cerrillo con un $10.37 \%$. Se registró también el coatín, el venado de cola blanca, La torcaza, el cuzumbo, la danta, el paujil, el mico churuco, el manao, el mico maicero, el puerco espín, el tintín, la guacharaca y el perro de monte.

Teniendo en cuenta el decreto nacional 1912 de 2017, se reportaron 15 especies sin categoría de amenaza, tres especies Vulnerables (VU) y una en estado crítico (CR); Para la clasificación internacional de la IUCN, 13 estuvieron en preocupación menor (LC), en la categoría internacional de UICN (LC), cinco en estado vulnerable (VU) y una cerca a la amenaza (NT).

\section{DISCUSIÓN}

Los trabajos con comunidades campesinas para evaluar la fauna con valor cinegético que habita en sus territorios, son limitados, más aún para esta zona puntual del piedemonte amazónico, sin embargo, en estudios realizados sobre el diagnóstico de comercio de carne silvestre, entre diversos grupos humanos, para otras zonas del piedemonte amazónico (5) reportan un total de 20 especies: 15 de mamíferos, 2 de aves y 3 de reptiles, en contraste con lo hallado en éste estudio, igual número de especies registradas pero de distintas clases, así: 17 mamíferos y 3 aves.

La guagua es una especie que se reporta como la más abundante, aquí y en el estudio del autor en mención. Ésta información cuantitativa permite tener un dato de partida en la riqueza de especies que están siendo cazadas en varios puntos del piedemonte, así como validar que los datos de este estudio hayan sido reportadas por comunidades campesinas de otros lugares para posteriores comparaciónes en áreas de mayor tamaño.

Otros estudios realizados en el flanco oriental del país $(6,7,8,9)$ sugieren que los ungulados son los mamíferos más cazados por comunidades indígenas y con mayor aporte en biomasa, lo que se diferencia de lo registrado en éste estudio en donde los roedores ocupan el mayor porcentaje de reporte, permitiendo inferir que existen varios factores (sociales, económicos y naturales) no comparados que influyan en las especies objetivo por cada uno de estos grupos humanos.

En este estudio Se encontró un total de 20 especies en contraste con la información institucional del PNNAFIW, en la cual se reportan 34 especies de mamíferos objeto de cacería y nueve especies de aves sin embargo, la toma de datos para éstos cálculos no fue sistemática, por lo que sugiere un sesgo en la información debido a la interpretación de imágenes utilizadas para la obtención de la información y no de una estructura de entrevista. De igual manera la información institucional coincide con que el armadillo (Dasypus c.f novemcinctus) y la boruga (Cuniculus paca) fueron las especies con más reportes de cacería (88.37\%), seguidas por el coatín (Dasyprocta fuliginosa) y el cerrillo (Tayassu pecari) con un porcentaje de $34.88 \%$ y $32.55 \%$ respectivamente.

El mico Churuco (Lagothrix lagotricha) se encuentra categorizado como Vulnerable a nivel nacional e internacional, lo que sugiere un cuidado importante y un proceso de investigación específico para medir el impacto de la cacería sobre este primate. si bien su frecuencia de aparición en los reportes de las encuestas fue bajo, no hay precisión sobre cuánta biomasa de esta especie está siendo consumida por las comunidades.

El Tigrillo (Leopardus tigrinus) se encuentra categorizado como vulnerable (VU) a nivel nacional e internacional, lo que conlleva a promover acciones de concienciación sobre la cacería de este felino. A pesar de que solo hubo un reporte de caza para este estudio y que su uso fue por control hacia los animales domésticos de la finca, es necesario implementar estrategias de conservación locales y/o articular a las nacionales.

La Danta Terrestre (Tapirus terrestris) se encuentra en estado crítico (CR) a nivel nacional y en estado vulnerable (VU) a nivel internacional, lo que sugiere un trabajo de concienciación y de fortalecimiento a la conservación de las dantas en el lugar. Es importante la articulación con el Plan Nacional de Dantas para Colombia a través de proyectos de investigación y alianzas interinstitucionales, ya que el piedemonte amazónico es un ecosistema importante en los procesos biológicos de la especie y puede verse afectada por una cacería sin control.

Para el caso del Cerrillo (Tayassu pecari), cuya distribución abarca parte de Centro América y sur América, se registró la categoría de Vulnerable (VU) a nivel internacional, pero sin reportes en la legislación nacional. este dato sugiere que 
Colombia presenta unas poblaciones más estables (en número de ejemplares para la especie) que deben promoverse en su conservación para que el impacto internacional mejore, sie y se estabilicen las poblaciones.

En conclusión, la fauna cinegética del PNNAFIW en los sectores (cuencas): Fragua Grande, Fragua Chorroso y Pescado está compuesta por 20 especies, tres de la clase Aves y 17 de la clase Mammalia, datos preliminares que sirven como línea base del conocimiento y requieren de un esfuerzo mayor de investigación para observar patrones de uso y grado de afectación poblacional de la fauna silvestre cinegética.

Se destacó el aporte al conocimiento de este proceso, enfocado a las dinámicas de los campesinos en el uso de fauna silvestre, situación que por lo general es publicada con comunidades indígenas y que es menester mostrar para el manejo acertado de las realidades de comunidades al interior de áreas protegidas del país.

\section{Conflicto de intereses}

No existen conflictos de intereses.

\section{Agradecimientos}

Al Grupo de Estudios en geología, Ecología y Conservación (GECO) de la Universidad del Cauca y al Parque Nacional Natural Alto Fragua Indi Wasi por la articulación institucional, a Edwin García Jaramillo por el acompañamiento en campo y asesoría, a Andrés Mauricio Bravo por la elaboración del mapa del área de estudio y a la comunidad campesina por su disposición e información suministrada.

\section{REFERENCIAS}

1. Hernández-López A, López-Alamilla E, Rodríguez-Ramírez A, Aquino-Bravata. Diagnóstico del Uso de la Fauna Silvestre en el Área de Protección de Flora y Fauna "Cañón del Usumacinta". Ra Ximhai. 2013; 9(1):1-13. http:// uaim.mx/webraximhai/Ej-26earticulosPDF/01-DiagnosticodelUsodelaFaunaSilvestreAlfredo.pdf

2. Ojasti J, Dallmeier F. Manejo de Fauna Silvestre Neotropical. Smithsonian Institution/MAB Biodiversity Program. Washington D.C; 2000. https://bibliotecavirtualaserena.files.wordpress.com/2017/11/libro-de-manejo-de-faunade-ojasti.pdf

3. Golden C,LiaF,BrasharesJ,RasolofoniainaB, KremenC.Benefits of wildlife consumptiontochildnutrition in biodiversity hotspot. Proc Natl Acad Sci USA. 2011; 108(49):19653-19656. https://dx.doi.org/10.1073\%2Fpnas.1112586108

4. Matallana C, Lasso CA, Baptiste MP. Carne de monte y consumo de fauna silvestre en la Orinoquia y Amazonia (Colombia y Venezuela). Instituto de Investigaciones de Recursos Biológicos Alexander von Humboldt, Universidad Nacional de Colombia: Bogotá D.C; 2012. http://repository.humboldt.org.co/bitstream/handle/20.500.11761/31376/213. pdf?sequence=1\&isAllowed $=y$

5. Quiceno-M MP, van Vliet N, Moreno J, Cruz-A D. Diagnóstico sobre el comercio de carne de monte en las ciudades de Colombia. CIFOR Documentos Ocasionales Indonesia. 2015; 1:58. https://www.cifor.org/publications/pdf files/ OccPapers/OP-136.pdf

6. Martínez-Salas MP, López-Arévalo HF, Sánchez Palomino P. Cacería de subsistencia de mamíferos en el sector oriental de la Reserva de Biósfera El Tuparro, Vichada (Colombia). Acta Biol Colomb. 2016; 21(1):151-166. https://www. crossref.org/iPage?doi=10.15446\%2Fabc.v21n1.49882

7. Plata A. Uso y percepción de la fauna silvestre en la cultura Sikuani, comunidad de Cumariana, Selva de Matavén, Vichada. [Tesis]. Bogotá: Departamento de Ecología y Territorio, Facultad de Estudios Ambientales y Rurales, Pontificia Universidad Javeriana; 2006. https://www.usergioarboleda.edu.co/wp-content/uploads/2012/06/ fauna-sivestre-vichada-colombia.pdf 
8. Ramos-Roca E. No sólo de plantas vive el hombre". Patrimonio alimentario y culinario y biodiversidad animal". Boletín de Antropología. Universidad de Antioquia, Medellín. 2019; 34(58):158-184. http://dx.doi.org/10.17533/ udea.boan.v34n58a09

9. Morales R, Díaz J. Uso de Fauna Silvestre Cinegética en la Comunidad de la Vereda El Zarzal, Serranía de los Churumbelos, Municipio de Mocoa, Departamento del Putumayo. Corpoamazonía y Universidad del Tolima; 2005. https://www.sinchi.org.co/files/PUBLICACIONES\%20DIGITALES/Zonificaci\%C3\%B3n\%20Ambiental\%20y\%20 Ordenamiento\%20de\%20la\%20Reserva\%20Forestal\%20de\%20la\%20Amazonia/4.\%09Fase\%20III\%3A\%20 Departamentos\%20de\%20Putumayo\%2C\%20Cauca\%2C\%20Nari\%C3\%B10\%20y\%20Meta/light4\%20F3\%20 Vol\%204\%20Estado\%20del\%20Arte\%20RFA\%20PNCM.pdf 